\title{
TTR
}

Traduction, terminologie, rédaction

\section{Kieran O'Driscoll. Retranslation through the Centuries: Jules Verne in English. Berne, Suisse, Peter Lang, 2011, 286 p.}

\section{Marie-France Guénette}

Volume 26, numéro 1, 1er semestre 2013

URI : https://id.erudit.org/iderudit/1036960ar

DOI : https://doi.org/10.7202/1036960ar

Aller au sommaire du numéro

\section{Éditeur(s)}

Association canadienne de traductologie

\section{ISSN}

0835-8443 (imprimé)

1708-2188 (numérique)

Découvrir la revue

Citer ce compte rendu

Guénette, M.-F. (2013). Compte rendu de [Kieran O’Driscoll. Retranslation through the Centuries: Jules Verne in English. Berne, Suisse, Peter Lang, 2011, 286 p.] TTR, 26(1), 265-271. https://doi.org/10.7202/1036960ar d'utilisation que vous pouvez consulter en ligne.

https://apropos.erudit.org/fr/usagers/politique-dutilisation/ 
in their evolution, translation is "constitutive" of the Americas (p. 5). Furthermore, his work corroborates both his call for and his anticipation of a new turn in translation studies- the socialpsychological turn, which "expand[s] a functional approach to include social effects and individual affects" (p.180). In doing so, he points out which research areas require further attention, thereby laying the groundwork for the eventual surge in this anticipated new turn.

Trish Van Bolderen
University OF OtTaWa

Kieran O'Driscoll. Retranslation through the Centuries: Jules Verne in English. Berne, Suisse, Peter Lang, 2011, 286 p.

Retranslation through the Centuries est la plus récente publication de Kieran O'Driscoll. Il s'agit d'un remaniement de sa thèse de doctorat, présentée à la Dublin City University en 2010. O'Driscoll se spécialise en histoire de la traduction ainsi qu'en études diachroniques des retraductions de l'œuvre de Jules Verne. Il est traducteur littéraire du français vers l'anglais et associé au projet de traduction des œuvres de Verne vers l'anglais de la North American Jules Verne Society.

Le livre présente une étude des retraductions vers l'anglais du roman Le tour du monde en quatre-vingts jours de Verne paru en 1873. Létude se veut un essai de méthodologie transhistorique, une nouveauté pour les chercheurs en histoire de la traduction, qui vise à rassembler plusieurs (re)traductions parues en Angleterre et aux États-Unis sur une période de 131 ans, soit de 1873 à 2004. O'Driscoll présente une analyse de la première traduction vers l'anglais du Tour du monde en quatre-vingts jours ainsi que de cinq retraductions. Dans cet effort, il étudie une multitude de facteurs connexes, tels les renseignements biographiques des traducteurs et les enjeux de publication des retraductions.

Le livre est divisé en trois parties. Au chapitre 1, O'Driscoll présente les fondements théoriques de sa recherche, les faits historiques entourant l'œuvre et la carrière de Verne, les éléments pertinents liés à la réception du roman Le tour du monde en quatre- 
vingts jours ainsi que la méthodologie mise en œuvre dans l'étude. Les chapitres 2 à 7 comportent une analyse détaillée de chacune des six traductions anglaises du Tour $d u$ monde sélectionnées par O'Driscoll, soit la première traduction par Towle (1873) et les retraductions de White (1874), Desages (1926), Baldick et Baldick (1968), Butcher (1995) et Glencross (2004). Chaque chapitre fournit les renseignements nécessaires à l'analyse descriptive des (re)traductions selon les critères présentés par Anthony Pym dans Method in Translation History (1998). O'Driscoll analyse les causes ayant influencé la production des traductions et synthétise les interactions entre elles. Le dernier chapitre réunit les conclusions de l'auteur en évoquant les objectifs de la recherche et le processus de sélection du corpus. Une bibliographie et un index thématique qui classe notamment les noms des théoriciens dont les propos ont été cités complètent le volume.

O'Driscoll véhicule l'idée principale suivante: en combinant l'étude diachronique descriptive des retraductions et de leurs traducteurs à l'étude de la réception des œuvres, il est possible d'expliquer les variations textuelles qui s'observent dans les retraductions d'une œuvre. Une telle approche permet en outre d'examiner l'influence des traducteurs dans la production des textes et dans les choix de traductions qui ont été faits. Ainsi, selon O'Driscoll, l'approche transhistorique est essentielle pour mener l'analyse de retraductions, car elle permet de juxtaposer les éléments textuels et les données biographiques relatives aux traducteurs. Ces données biographiques sont d'une grande importance afin de tirer des conclusions sur la société dans laquelle les textes ont été rédigés. Dans les mots de Pym: "[f]or almost every inner causation that one finds in a translator's personal biography there is a wider, social mode of causation that enables or accepts inner factors to leave their mark in the public world of translations » (cité par O'Driscoll, 2011, p. 17).

Selon O'Driscoll, les retraductions ne s'inscrivent pas dans une trajectoire linéaire de progrès, mais sont plutôt produites pour des raisons très différentes et dans des contextes historiques particuliers. En analysant les multiples causes entourant le projet et le processus de traduction, O'Driscoll peut expliquer pourquoi certaines traductions se ressemblent énormément, alors que d'autres diffèrent les unes des autres. Il cherche à répondre aux questions 
suivantes: qui a traduit quoi, quand, pourquoi et pour quelles raisons? En se renseignant sur les traductions et les traducteurs qui les ont effectuées, O'Driscoll cherche à clarifier les enjeux de production et à déduire les normes sociales et culturelles qui ont influencé les textes cibles. Il tente également d'expliquer la réception du roman de Verne dans les cultures réceptrices anglophones de l'Angleterre et des États-Unis. Par le biais d'une analyse des causalités de la traduction, il vise à cerner pourquoi certaines traductions ont été bien reçues, tandis que d'autres ont été rapidement oubliées.

L'auteur se réclame du courant de pensée des Descriptive Translation Studies (DTS) de Gideon Toury. Il propose une étude descriptive des (re)traductions expliquées au moyen d'une série de facteurs connexes. Sa recherche s'inscrit aussi dans la lignée des travaux de Pym (1998), qui s'intéresse aux interventions des traducteurs, à leur influence sur la production des textes ainsi qu'à leur présence dans les traductions. Pour construire sa méthodologie, O'Driscoll utilise principalement les notions des études descriptives de Toury (1995) et celles de Pym (1998) sur l'histoire de la traduction et les causalités en traduction.

L'auteur compare le texte source et les textes cibles tout en considérant l'analyse des données biographiques disponibles sur les traducteurs, de façon à pouvoir déterminer l'effet des circonstances personnelles et professionnelles des traducteurs sur les textes cibles. En évaluant la perception de la traduction qu'ont les traducteurs, O'Driscoll tente d'évaluer l'influence de cette perception sur leurs décisions traductionnelles. Ce faisant, il parvient à dégager l'effet des causes externes aux traducteurs, telles les contraintes linguistiques et les normes sociales et culturelles régissant le travail de traduction. Pour organiser les données recueillies, O'Driscoll a construit des tableaux selon les catégories interprétatives proposées par Pym (1998). Les quatre catégories, ou causes, repérées sont 1) matérielles, 2) finales, 3) formelles et 4) efficientes. Elles aident respectivement à déceler: 1) l'influence des ressources linguistiques de la langue source et la langue cible; 2) l'influence des actants des réseaux de traduction; 3) l'influence des normes initiales, préliminaires et opérationnelles, telles que définies par Toury (1995); 4) l'influence du traducteur comme agent libre dans sa création du texte cible. 
Concernant l'établissement de son corpus d'étude, l'auteur a recensé les douze traductions vers l'anglais du roman de Verne parues entre 1873 et 2004. Les retraductions analysées devaient être représentatives du projet d'étude transhistorique et distribuées équitablement sur la période détude. O'Driscoll a donc pris soin d'inclure la première traduction ainsi que la dernière retraduction. Il dit avoir utilisé une méthode d'échantillonnage aléatoire pour sélectionner les quatre autres retraductions, mais précise par ailleurs qu'il les a choisies en fonction de la richesse des renseignements biographiques disponibles sur les traducteurs et des annotations paratextuelles. Ces derniers facteurs tendent ainsi à déconstruire l'argument de l'échantillonnage aléatoire.

Pour ce qui est de la sélection des éléments du roman de Verne à analyser, O'Driscoll a retenu le premier et le dernier chapitre (chapitres 1 et 37) de même que le métatexte et le paratexte, les notes explicatives et les glossaires. Il a également procédé à une analyse comparative de cinq autres chapitres, soit les chapitres 11 , $16,18,30$ et 35 , choisis aléatoirement. Son étude compare des segments de l'original avec les segments correspondants dans le texte cible. O'Driscoll a évalué le degré de déviance des retraductions par rapport au texte original, calculé selon le nombre de métamorphoses (shifts, v. Chesterman, 2000) recensées. Les autres chapitres du roman ont été sélectionnés selon leur pertinence dans le cadre de la recherche. L'auteur précise que ces choix sont nécessairement subjectifs, mais qu'ils servent à montrer la façon dont les traducteurs ont traduit un passage important du roman. Cet amalgame de méthodes d'échantillonnage que l'auteur qualifie d'aléatoire entraîne certaines ambiguïtés dans la sélection du corpus.

Dans son étude, O'Driscoll présente trois hypothèses connexes au statut littéraire de Verne qui reflètent son utilisation du modèle à causes multiples. Il postule que le manque de prestige littéraire de l'œuvre de Jules Verne en France explique la faible qualité des premières traductions. Selon lui, le changement de statut des œuures de Verne aurait mené à une amélioration du calibre des (re)traductions vers l'anglais du Tour du monde. Il suppose une corrélation entre l'inclusion d'un roman de Verne dans les épreuves de l'agrégation en France dans les années 1960 et lélévation du statut littéraire de ses œuvres dans le monde 
littéraire anglophone. L'élévation du statut littéraire de Verne en France aurait donc mené à une amélioration du calibre et de la fidélité des traductions de son œuvre vers l'anglais.

O’Driscoll dresse également un parallèle entre la réception de l'œuvre de Verne dans les pays anglophones et le calibre des traductions. Vers 1873, Verne avait en Angleterre la réputation d'être un «auteur pour garçons», alors qu'aux États-Unis, les critiques le proclamaient «Prince of the Genre of the Fantastic in Literature»(Evans, 2005, p. 33, cité à la p. 40). L'œuvre de Verne aurait été accueillie positivement aux États-Unis parce que celui-ci incorporait des éléments de la conscience collective américaine dans ses textes. Dans Le tour $d u$ monde, il y a de nombreuses descriptions de voyages en train, et deux chapitres décrivent une bataille entre des Blancs et des Indiens sioux. Puisque la construction du chemin de fer était d'actualité et qu'il y avait des conflits fréquents entre les Blancs et les Indiens en Amérique, le texte de Verne devenait presque une concrétisation de l'imaginaire collectif. De plus, O'Driscoll avance que la guerre civile et les vagues d'immigration ont engendré un faible taux d'alphabétisation chez les Américains. Les textes de Verne étant jugés pédagogiques et divertissants, ils auraient bénéficié du soutien de l'État, qui appuyait les initiatives de lecture et l'instruction de son peuple. O'Driscoll mentionne donc diverses avenues possibles pour expliquer les différences observées quant à la réception du Tour du monde en France, en Angleterre et aux États-Unis. Le contraste entre l'hypothèse de l'agrégation en France et la réception de l'œuvre de Verne en Amérique gagnerait toutefois à être développé, car le lecteur se retrouve devant une multitude de réponses possibles, sans aucune certitude que l'une est plus plausible qu'une autre. D'ailleurs, O'Driscoll revient sur la causalité des résultats de traduction à la fin de son ouvrage et précise que l'attribution de la causalité était spéculative et que les constats de son étude ne sont que des influences de traduction quasi causales (p. 262).

O'Driscoll affirme que les retraductions vers l'anglais du Tour $d u$ monde en quatre-vingts jours sont mieux expliquées avec un modèle de causes multiples qu'avec l'hypothèse de la retraduction de Berman (1990) selon laquelle une retraduction est généralement plus sourcière qu'une première traduction. Pour O'Driscoll, 
ce modèle est trop simpliste, car il ne prend pas en compte les causes expliquant la raison d'être des retraductions. Le modèle est d'ailleurs insuffisant dans le cas du roman de Verne, où il est difficile de repérer une seule traduction ayant été perçue comme un texte canonique. Au terme de son étude, O'Driscoll conclut ainsi que les (re)traductions ne s'améliorent pas constamment en devenant davantage sourcières que ciblistes, mais dépendent plutôt d'un processus complexe qui est à la fois humain, social et politique.

L'auteur allègue aussi que le traducteur est un agent important qui a le pouvoir d'influencer la forme du texte cible. Il stipule que les traducteurs dont il a étudié les textes ont montré leur capacité à changer le texte de cinq manières: en utilisant un idiolecte traductionnel unique, en corrigeant des inexactitudes, en réinterprétant des thèmes, en inscrivant des commentaires paratextuels et en laissant transparaître leurs intérêts personnels dans la traduction. Pour O'Driscoll, les différences mises en lumière dans les retraductions anglaises du roman sont dues en partie à l'interférence des traducteurs dans la transmission du texte et non à une obéissance à des normes sociales et culturelles. L'auteur montre également qu'il y a moyen d'expliquer les enjeux de traduction en considérant la parution de traductions comme un processus complexe et indéterministe.

Les apports de cette analyse sont, entre autres, une innovation méthodologique des études de cas transhistoriques et une remise en cause réussie de l'hypothèse du progrès en retraduction d'Antoine Berman (1990). De plus, l'auteur apporte une contribution importante à l'étude des traductions vers l'anglais des œuvres de Jules Verne, dont le corpus n'a pas été amplement analysé jusqu'ici. Pour ce qui est des apports d'ordre méthodologique, O’Driscoll présente une approche qui intègre différents aspects des DTS et qui inspire les chercheurs à emprunter les idées de nombreux théoriciens pour contourner les difficultés méthodologiques encourues dans des projets de recherche de grande envergure. L'auteur ne traite malheureusement pas de l'importance des relations entre les éditeurs et les traducteurs dans les choix de traduction, tel qu'il indique vouloir le faire dans l'introduction. 


\section{Références}

BERMAN, Antoine (1990). "La retraduction comme espace de la traduction». Palimpsestes, 4, p. 1-7.

CHESTERMAN, Andrew (2000). Memes of Translation: The Spread of Ideas in Translation Theory. Amsterdam/Philadelphia, John Benjamins.

EVANS, Arthur B. (2005). «Jules Verne's English Translations». ScienceFiction Studies, 32, 1, p. 80-104.

PYM, Anthony (1998). Method in Translation History. Manchester, St Jerome Publishing Ltd.

TOURY, Gideon (1995). Descriptive Translation Studies and Beyond. Amsterdam/Philadelphia, John Benjamins.

Marie-France Guénette Université De MontréAL 